\title{
Multiuser Detection using IDMA Scheme in UWB Home Environment
}

\author{
Vishal Shukla \\ Department of Electronics \\ Engineering \\ Harcourt Butler Technological \\ Institute Kanpur- 208002, India
}

\author{
Manoj Kumar Shukla \\ Department of Electronics \\ Engineering \\ Harcourt Butler Technological \\ Institute Kanpur- 208002, India
}

\author{
Tanuja Pande \\ Department of Electronics \\ Engineering \\ Harcourt Butler Technological \\ Institute Kanpur- 208002, India
}

\begin{abstract}
Due to inherent advantages of high speed transmission, immunity towards multipath and low cost, ultra wide band (UWB) has become the key technology for the next generation of wireless communication systems. This paper addresses the performance of Interleave Design Multiple Access scheme (IDMA), in absence of any narrow band interference. Simulations are performed using channel sounding of the $2-8 \mathrm{GHz}$ band collected in the residential scenario and characterized by high level of multipath components. This paper shows the performance of IDMA scheme for different users in multiple access interference environments.
\end{abstract}

\section{General Terms}

UWB channel models, Multiple Access Interference, CDMA (Code Division Multiple Access), BER (Bit Error Rate) etc.

\section{Keywords}

IDMA (Interleave Design Multiple Access), chip by chip iterative detection etc.

\section{INTRODUCTION}

Ultra-Wideband technology is an emerging technology which has attracted the researchers to consider it as the future technology for the short range communication Historically, UWB radar systems were developed mainly as a military tool because they could 'see through' trees and beneath ground surfaces. However, recently, UWB technology has been focused on consumer electronics and communications. Ideal targets for UWB systems are low power, low cost, high data rates, precise positioning capability, and extremely low interference.

As UWB is a new technology so it should not disturb the other existing narrow band technology which is already has been deployed, so UWB systems are necessarily required to operate in the existence of strong multiple interferences [4],[5].

Today, Ultra Wideband wireless communication technology has become a hot cake for researchers for transmitting a large amounts of digital data over a wide frequency spectrum using very short duration pulses, low powered radio signals [4], [7]. IR (Impulse radio), a form of UWB spread spectrum signalling technique is used for short-range applications in a dense multipath environment [5], [6]. An impulse radio technique is that technique which is carrier free now the question comes, why it is carrier free? The to this question is that IR (impulse radio) technology does not involve any use of carrier for transmitting and receiving data, in this technology the data/information is send by short duration pulses which are carrier less [11], [12].

Ultra-wideband (UWB) radio is an emerging technology in WPAN wireless systems that have attracted a great interest from academia, industries, and global standardization bodies.

Within the 802.15 working group (WG) there are two task groups(TGs) IEEE 802.15.3a (TG3a) and IEEE 802.15.4a (TG4a) that develop their standards based on UWB technology.

As we all are aware of the power of CDMA (Code Division Multiple Access Scheme) to detect multiple users. Another variant of CDMA is IDMA in which users are distinguished by different interleaving patterns rather than user specific spreading codes as in the case of CDMA.

Here in this paper the performance of IDMA scheme in terms of BER (Bit Error Rate), in different UWB channel models has been shown in multiple user environment.

\section{IDMA SCHEME}

In communication systems, interleaving is referred to be technique commonly used to overcome correlated channel noise such as burst error or fading [3]. In interleaving mechanism, the input data rearranges itself such that consecutive data bits are split among different blocks and is swapped in a known pattern amongst them. At the receiver end, the interleaved data is arranged back into the original sequence with the help of de-interleaver. As a result of interleaving, correlated noise introduced in the transmission channel appears to be statistically independent at the receiver and thus allows better error correction.

The user-specific interleavers play vital role in the efficiency of IDMA system. It not only provides decorrelation between adjacent bit sequences as provided in the case of orthodox turbo coding and decoding, but also facilitates a means for decorrelating various users [1]. The correlation between interleavers should measure how strongly signals from other users affect the decoding process of a specific user. The decorrelation among the user- specific interleavers provides a mean to reduce the multiple access interference (MAI) from other users thus helping in the convergence of detection process.

\subsection{IDMA Transmitter and Receiver}

A simple IDMA scheme transmitter and receiver structure is shown in the Figure 1. 


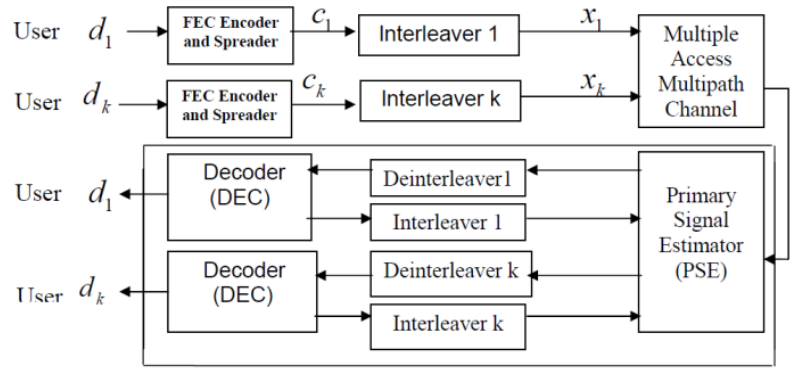

Fig 1: IDMA Transmitter \& Receiver Structure

The upper part of Figure 1 demonstrates the transmitter structure of the IDMA scheme with $\mathrm{K}$ simultaneous users present in the system. The input data sequence of user-k is encoded based on a low-rate code $\mathrm{C}$, generating a coded sequence,

$$
C_{k}=\left[c_{k}(1), c_{k}(2), \ldots c_{k}(j) \ldots . c_{k}(J)\right]^{T}
$$

where $\mathrm{J}$ is the frame length. The elements in $\mathrm{c}_{\mathrm{k}}$ are referred to as coded bits. Then $c_{\mathrm{k}}$ is permutated by an interleaver $\pi_{\mathrm{k}}$, so, producing

$X_{k}=\left[x_{k}(1), x_{k}(2), x_{k}(3), \ldots x_{k}(j) . . x_{k}(J)\right]^{T} . \quad$ Following the CDMA convention, the element in $k x$ will be denoted as "chips". Users are solely distinguished by their interleavers, hence the name interleave division multiple access (IDMA) scheme [3].

The key principle of IDMA is that the interleavers $\left\{\pi_{k}\right\}$, opted for user separation, and should be orthogonal for all the users. It is assumed that the interleavers are generated independently and randomly. The randomly generated interleavers disperse the coded sequences so that the adjacent chips are approximately uncorrelated, facilitating the simple chip-bychip detection scheme as discussed below. Adopting an iterative sub-optimal receiver structure, as demonstrated in figure 3.6, consisting of the primary signal estimator (PSE) and $\mathrm{K}$ single user a posteriori probability (APP) decoders (DECs), the data is iterated for pre-decided number iterations before finally taking hard decision on it. For single path propagation, as shown in figure 2.4, there is only one path for the transmission. The multiple access and coding constraints are considered separately in the PSE and DECs. The outputs of the PSE and DECs are extrinsic log-likelihood ratios (LLRs) about $\left\{x_{k}(j)\right\}$ defined below as;

$$
e\left(x_{k}(j)\right)=\log \left(\frac{p\left(y \mid x_{k}(j)\right)=+1}{p\left(y \mid x_{k}(j)\right)=-1}\right), \quad \forall K, j
$$

Those LLRs are further distinguished by subscripts, i.e. $e_{P S E}\left(x_{k}(j)\right)$ and $e_{D E C}\left(x_{k}(j)\right)$, depending on whether they are generated by the PSE or DECs.

For the PSE section, $\boldsymbol{y}$ in (2) denotes the received channel output while for the DECs, $\boldsymbol{y}$ in (2) is formed by the deinterleaved version of the outputs of the primary signal estimator (PSE) block. A global turbo type iterative process is then applied to process the LLRs generated by the PSE and DECs blocks [3].

\subsection{Basic Primary Estimator (PSE)}

Assuming that the channel with no memory and after chip matched filtering, the received signal from $\mathrm{K}$ users can be written as;

$$
r(j)=\sum_{k=1}^{K} h_{k} x_{k}(j)+n(j), \quad j=1,2, . . \mathrm{J}
$$

where $h_{\mathrm{k}}$ is the channel coefficient for user-k and $\{\mathrm{n}(\mathrm{j})\}$ are samples of an AWGN process with zero mean and variance, $\sigma^{2}=N_{0} / 2$. Assuming that the channel coefficient $\left\{h_{\mathrm{k}}\right\}$ are known a priori at the receiver. Due to the use of random interleaver $\left\{\pi_{\mathrm{k}}\right\}$, the PSE operation can be carried out in a chip-by-chip manner, with only one sample r(j) used at a time.

$$
\begin{gathered}
r(j)=h_{k} x_{k}(j)+\xi_{k}(j) \\
\xi_{k}(j)=r(j)-h_{k} x_{k}(j)=\sum_{k^{\prime} \neq k} h_{k^{\prime}} x_{k^{\prime}}(j)+n(j)
\end{gathered}
$$

where, $\xi_{\mathrm{k}}(\mathrm{j})$ is the distortion (including interferenceplus-noise) in $\mathrm{r}(\mathrm{j})$ with respect to user-k. From the central limit theorem $\xi_{\mathrm{k}}(\mathrm{j})$, can be approximated as a Gaussian variable, and $r(j)$ can be characterized by a conditional Gaussian probability density function;

$$
\begin{aligned}
& p\left(\frac{r(j)}{x_{k}(j)}= \pm 1\right) \\
& =\frac{1}{\sqrt{2 \pi \operatorname{Var}\left(\xi_{k}(j)\right)}} \exp \left(-\frac{\left(r(j)-\left( \pm h_{k}+E\left(\xi_{k}(j)\right)\right)\right)^{2}}{2 \operatorname{Var}\left(\xi_{k}(j)\right)}\right)
\end{aligned}
$$

where, E (.) and Var (.) are the mean and variance functions, respectively.

\subsection{Algorithm for chip by chip Detection}

Step (i): Estimation of Interference Mean and Variance

$$
\begin{gathered}
E(r(j))=\sum_{k} h_{k} E\left(x_{k}(j)\right), \\
\operatorname{Var}(r(j))=\sum_{k}\left|h_{k}\right|^{2} \operatorname{Var}\left(x_{k}(j)\right)+\sigma^{2}, \\
E\left(\xi_{k}(j)\right)=E(r(j))-h_{k} E\left(x_{k}(j)\right) \\
\operatorname{Var}\left(\xi_{k}(j)\right)=\operatorname{Var}(r(j))-\left(\left|h_{k}\right|^{2} \operatorname{Var}\left(x_{k}(j)\right)\right.
\end{gathered}
$$

Step (ii): LLR Generation

$$
e_{P S E}\left(x_{k}(j)\right)=2 h_{k} \cdot \frac{r(j)-E\left(\xi_{k}(j)\right)}{\operatorname{Var}\left(\xi_{k}(j)\right)}
$$

\subsection{DEC Function}

The DECs in figure 1 carry out APP decoding using the output of the PSE as the input. With binary phase shift keying (BPSK) signaling, their output is the extrinsic log-likelihood ratios (LLRs) $e_{D E C}\left(x_{k}(j)\right)$ of $\mathrm{x}_{\mathrm{k}}(\mathrm{j})$ defined in (2), which are used to generate the following statistics,

$$
\begin{aligned}
& e_{D E C}\left(x_{k}(\pi(j))\right)=\sum_{j=1}^{S} e_{E S E}\left(x_{k}(\pi(j))\right) \\
& E\left(x_{k}(j)\right)=\tanh \left(\frac{e_{D E C}\left(x_{k}(j)\right)}{2}\right), \\
& \operatorname{Var}\left(x_{k}(j)\right)=1-\left(E\left(x_{k}(j)\right)\right)^{2}
\end{aligned}
$$

In the iterative process, PSE and DEC- $k$ exchange the extrinsic information about $x_{k}(j)$. The CBC detection for IDMA scheme can be concluded as follows, (1) Primary signal estimator generates $e_{P S E}\left(x_{k}(j)\right)$ by $(3.13 \mathrm{e})$ for decoder 
DEC- $k$. (2) DEC- $k$ generates $e_{D E C}\left(x_{k}(\pi(j))\right)$, which are used to update mean and variance of $x_{k}(j)$.

Under the assumption that $\left\{x_{k}(j)\right\}$ are independent, (7)-(11) are a straightforward consequence of (4) and (5). The Step (ii), shown in algorithm, is obtained by evaluating (2) based on (5). Algorithm shown is an extremely simplified form for all spreading sequences to be length-1. The operations in (7) and (8), i.e., generating $E(r(j))$ and $\operatorname{Var}(r(j))$, are shared by all users, costing only three multiplications and two additions per coded bit per user. Overall, the PSE operations shown in step (i) and step (ii), cost only seven multiplications and five additions per coded bit per user, which is very modest [3]. Interestingly, the cost per information bit per user is independent of the number of users $\mathrm{K}$. This is considerably lower than that of other alternatives.

\section{UWB Channel Models}

The accurate design of channel model is a very important issue for ultra wideband WPAN (Wireless Personal Area Network) communication system. Such a model creates the facility for calculation of large and small-scale characteristics [10]. Specially large-scale models are necessary for network planning and link budget design and small-scale models are necessary for efficient receiver design. The most famous multipath UWB indoor channel models are tap-delay line Rayleigh fading model, Saleh and Valenzuela (S-V) model and $\Delta-\mathrm{K}$ model. The measurements of $\mathrm{S}-\mathrm{V}$ channel shows that the multipath components arrive from transmitter to receiver in the form of clusters [11]. The different paths of such wide band signal can rise to several multipath components, all of which will be part of one cluster. The arrival of multipath components is modeled by Poisson distribution and thus the inter arrival time between multipath components follow based an exponential distribution. The multipath arrival of UWB signals are grouped into two categories: cluster arrival and ray arrival within a cluster. This model requires several parameters to describe indoor channel environments [28]. Ray arrival rate at which a multipath component arrive in a cluster. The cluster arrival rate is always smaller than the ray arrival rate. The amplitude statistics in $\mathrm{S}-\mathrm{V}$ model are based on lognormal distribution, the power of which is controlled by the cluster and ray decay factor [29]. Indoor channel environments are classified as CM1, CM2, CM3, and CM4 following IEEE 802.15.3a standard based on propagation conditions as follows [11]. According to [11], [13], the channel impulse response is defined as

$$
h(t)=X \sum_{l=0}^{L-1} \sum_{k 0}^{K-1} \alpha_{k} \delta\left(t-T_{l} \mid-\tau_{k, l}\right)
$$

where $\left\{\alpha_{\mathrm{k}, \mathrm{l}}\right\}$ a are the multipath gain coefficient of $k^{\text {th }}$ ray related to $l^{\text {th }}$ cluster, $\left\{\mathrm{T}_{1}\right\}$ is the delay or arrival time of first path of the $l^{\text {th }}$ cluster, $\left\{\tau_{\mathrm{k}, 1}\right\}$ is the delay of the $k^{\text {th }}$ multipath component within the $l^{\text {th }}$ cluster relative to arrival time $\left\{\mathrm{T}_{1}\right\},\{X\}$ denotes the lognormal shadowing term. Equation 14 shows the cluster arrival time and the ray arrival distribution time

$$
\begin{aligned}
& p\left(T_{l} \mid T_{l-1}\right)=\Lambda \exp \left[-\Lambda\left(T_{l}-T_{l}\right)\right], \quad l>0 \\
& p\left(\tau_{k, l} \mid \tau_{(k-1), l}\right)=\left[\lambda\left(\tau_{k, l}-\tau_{(k-1), l}\right)\right], \quad k>0
\end{aligned}
$$

where $\tau_{0,1}=0$. If the channel coefficients are considered as real, then $p_{k, l}$ takes real random value +1 or -1 . The channel coefficients are defined as:

$$
\alpha_{k, l}=p_{k, l} \xi_{l} \beta_{k, l}
$$

If complex base band channel is considered, the channel coefficient phase is uniformly distributed over the interval $[0$, $2 \pi]$, is the amplitude of the UWB signal. It is based on lognormal distribution and is given as

$$
\begin{aligned}
20 \log _{10}\left(\xi_{l} \beta_{k, l}\right) & \propto \operatorname{Normal}\left(\mu_{k, l}, \sigma_{1}^{2}+\sigma_{2}^{2}\right), \\
\left|\xi_{l} \beta_{k, l}\right| & =10^{\frac{\mu_{1}+\mu_{2}}{20}}
\end{aligned}
$$

where $\sigma_{1}$ is the standard deviation of cluster lognormal fading term, $\sigma_{2}$ is the standard deviation of ray lognormal fading term $\mathrm{n} 1$ and $\mathrm{n}_{2}$ are independent and correspond to the fading on each cluster and ray. The behavior of the averaged power delay profile is

$$
E\left[\left|\xi_{l} \beta_{k, l}\right|^{2}\right]=\Omega_{0} \mathrm{e}^{-\mathrm{T}_{1} / \Gamma} \mathrm{e}^{-\tau_{\mathrm{k}, 1} / \nu}
$$

Where, $\Omega_{0}$ is the mean energy of the first path of the first cluster. The $\mu_{k, l}$ is given by

$$
\mu_{k, l}=\frac{10 \ln \left(\Omega_{0}\right)-\frac{10 T_{l}}{\Gamma}-10 \tau_{k, l} / \Upsilon}{\ln (10)}-\frac{\left(\sigma_{1}^{22}+\sigma_{2}^{2}\right) \ln (10)}{20}
$$

Where, $\xi_{\mathrm{k}, \mathrm{l}}$ correspondence the fading associated with the $1^{\text {th }}$ cluster, and $\beta_{\mathrm{k}, \mathrm{l}}$ corresponds to the fading associated with the $k^{\text {th }}$ ray of the $l^{\text {th }}$ cluster. This reflects the exponential decay of each ray as well as decay of the total cluster power with respect to delay. $X$ is the shadowing term and it is characterized by following

$$
20 \log 10(X) \underset{\text { Normal }}{ }\left(0, \sigma^{2}\right)
$$

$\sigma$ is the standard deviation of lognormal shadowing term.

\subsection{Impulse Response of Different Channel Models}

This section shows the impulse responses of different four type channel models.

CM1 describes a line-of-sight (LOS) scenario with T-R separation less than $4 \mathrm{~m}$.

CM2 describes the same range for T-R separation but it is in NLOS (non line of sight).

CM 3 describes a NLOS medium for separation between transmitter and receiver of range $4-10 \mathrm{~m}$.

CM4 describes an environment of more than $10 \mathrm{~m}$ with strong delay dispersion. 


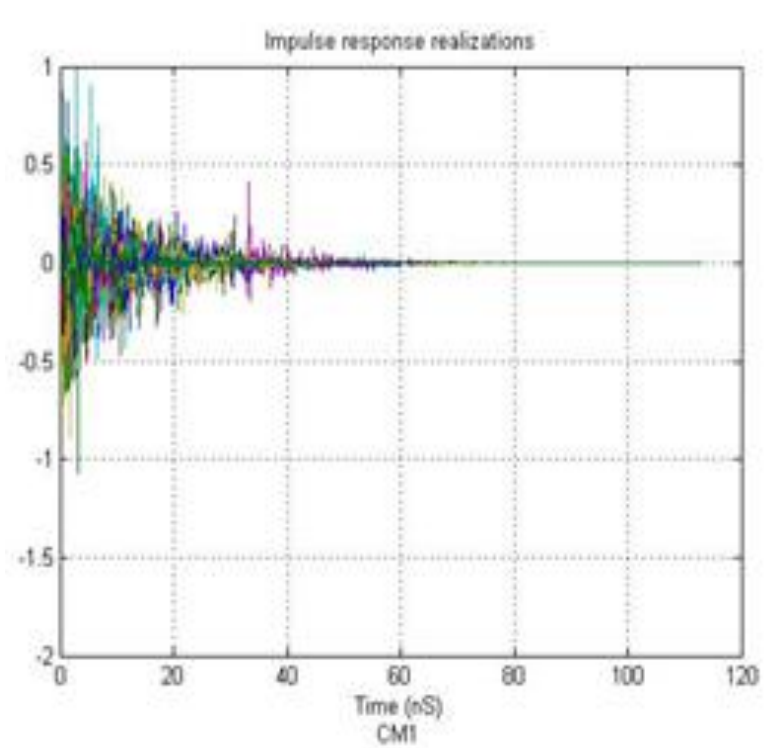

Fig 2: Impulse Response of CM1

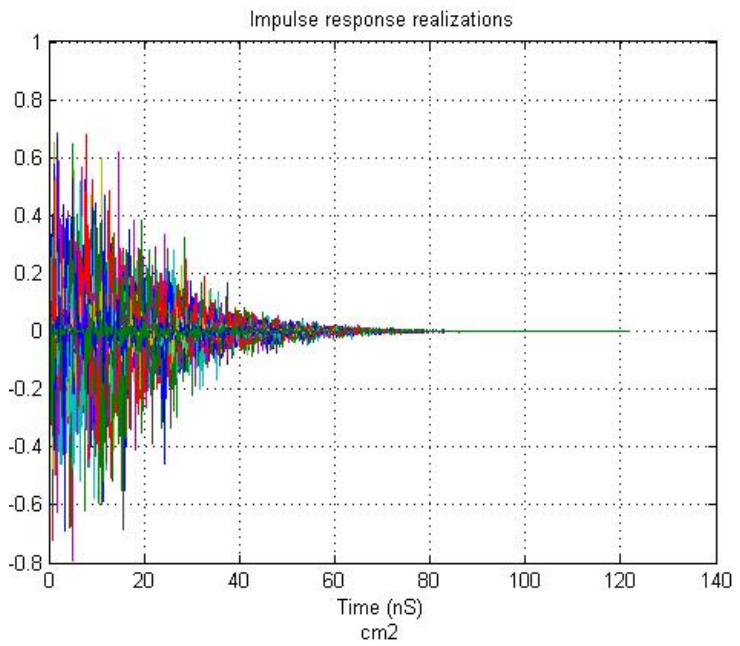

Fig 3: Impulse Response of CM2

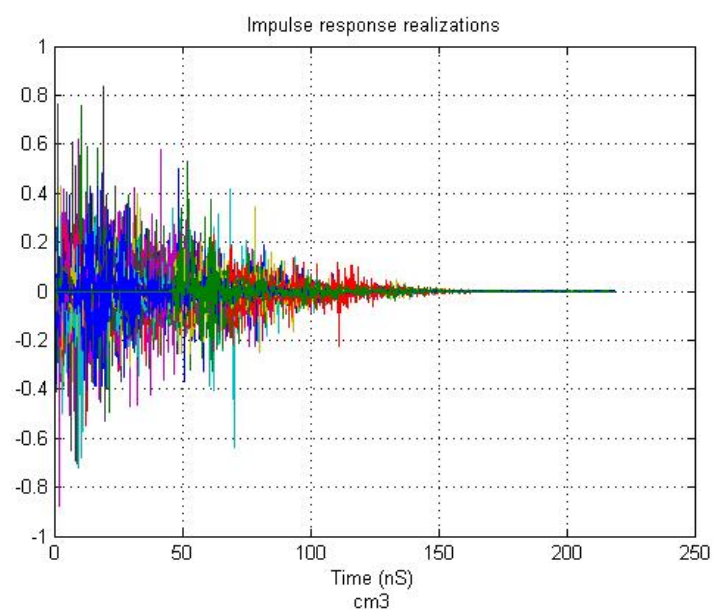

Fig 4: Impulse Response of CM3

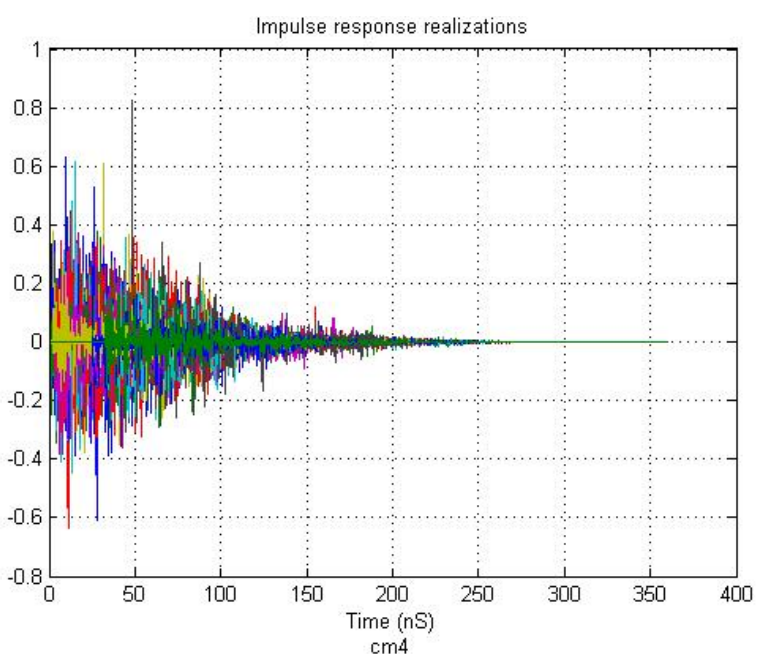

Fig 5: Impulse Response of CM4

\section{SIMULATION RESULTS}

In this section, BER performance of IDMA scheme is shown. These simulation results are shown using the parameters: spreading length 16 PN codes, total iteration 5, user count vary from 1 to 32, and Multiple Access Interference (MAI) has been issued here. These simulations are performed using channel sounding of $2-8 \mathrm{GHz}$ band collected in residential setting, for bit rates of $100 \mathrm{Mbps}$ and spreading codes of length 16, for all users present in the system. Figure 6 to 9 shows the BER performance of IDMA scheme for varying users in LOS (Line of Sight) and NLOS (Non Line of Sight) scenario.

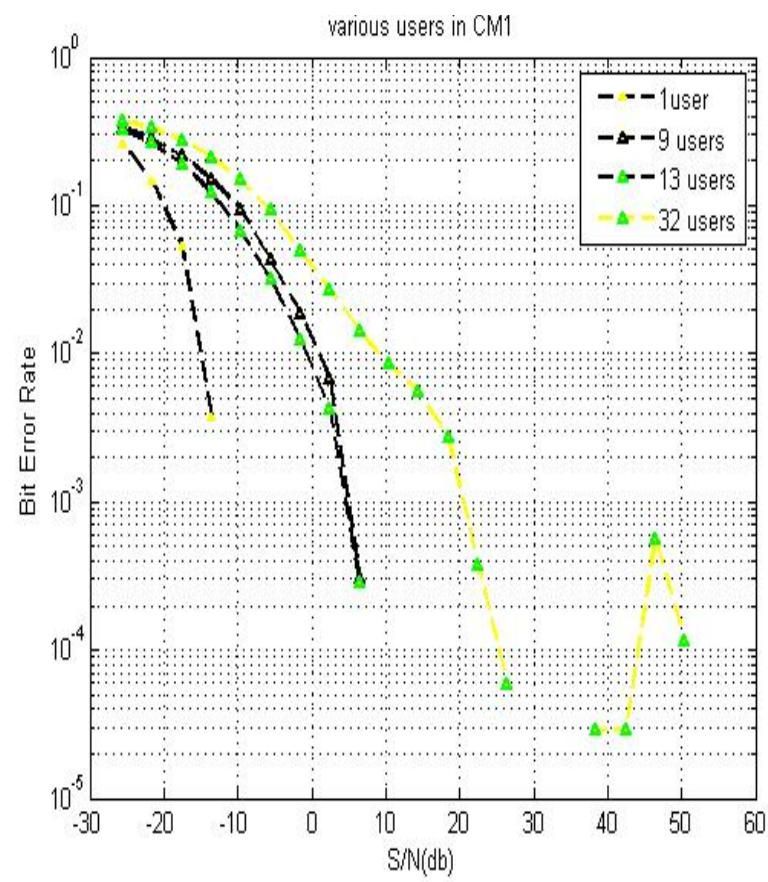

Fig 6: BER Performance of IDMA scheme in CM1 (LOS) 


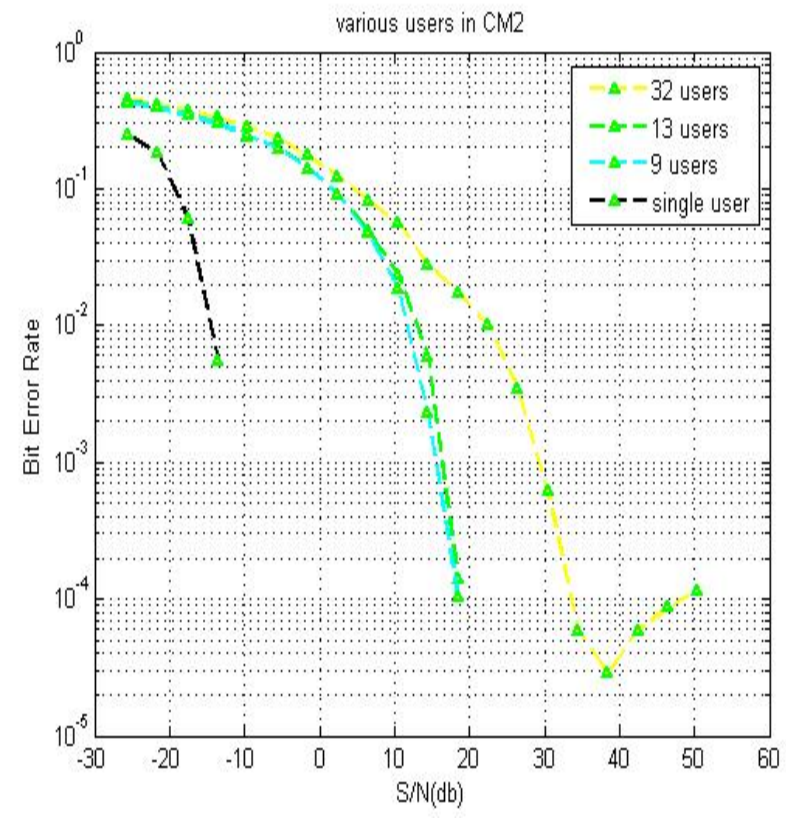

Fig 7: BER Performance of IDMA scheme in CM2 (NLOS)

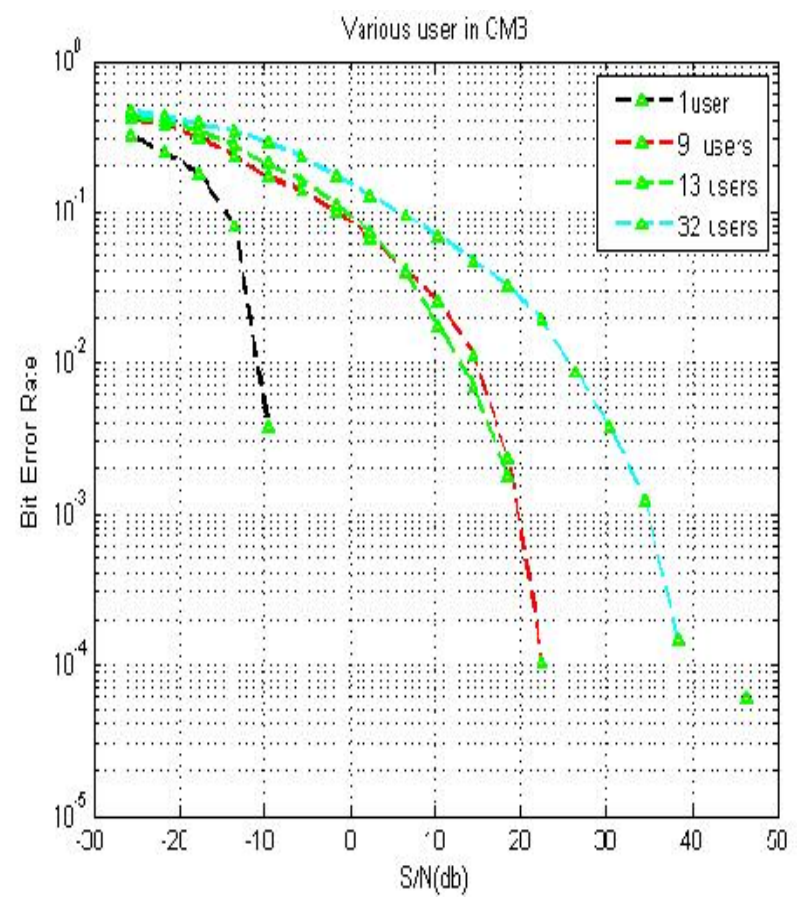

Fig 8: BER Performance of IDMA scheme in CM3 (NLOS)

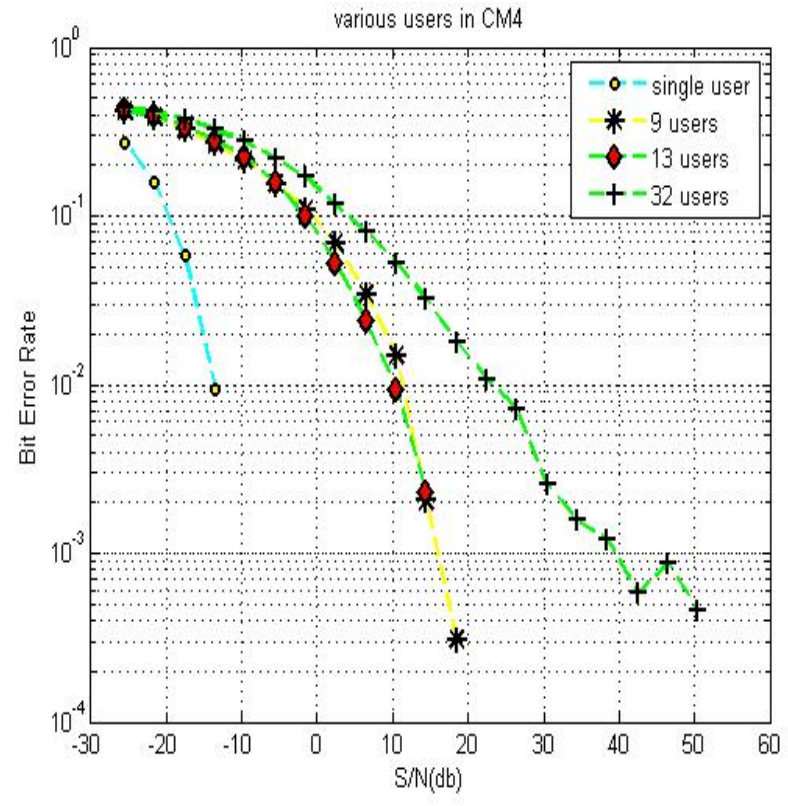

Fig 9: BER Performance of IDMA scheme in CM4 (NLOS)

\section{CONCLUSION}

This paper discusses the new emerging technology for the short range wireless communication systems, named UWB, at the beginning this paper contains the short description of UWB technology in which extremely short duration pulses are used to send the digital data and these pulses have bandwidth of order of several GHz.

Then a new variant of CDMA scheme which has certain advantages over CDMA is discussed in detail. The importance of CDMA scheme to detect multiple users in UWB home environment is addressed in [12]. Here in this paper it is shown that by using IDMA scheme in UWB home environment BER achieved is approximately $10^{-4}$ at lower value of signal to noise ratio (SNR). MAI and the effectiveness of new multiple access scheme IDMA is issued to detect multiple users in UWB indoor environment.

\section{REFERENCES}

[1]. Manoj Kumar Shukla, "Performance Evaluation of IDMA Scheme in Wireless Communication," PhD thesis, Nov. 2010.

[2] J. G. Andrews, "Interference cancellation for cellular systems: A contemporary overview," IEEE Trans. Wireless Commun., vol. 12, pp. 19-29, Apr. 2005.

[3] Li Ping, Lihai Liu, Keying Wu, W. Leung, "Interleave Division Multiple Access," IEEE Transactions on Wireless Communications, vol. 5, pp. 938-947, April 2006.

[4] C.Fowler, J.Entzminger, J.Corum, "Assessment of ultrawideband (UWB) technology," IEEE Aerospace and Electronics Systems Magazine, vol.5, no.11, pp.45-49, Nov. 1990.

[5] R.S Vickers, "Ultra-wideband radar-potential and limitations," IEEE MTT-S International Microwave Symposium Digest, vol.1, pp.371-374, 1991. 
[6] I.Jouny, "Wavelet decomposition of UWB radar signals," IEEE Antennas and Propagation Society International Symposium, vol.2, pp.1132-1135, 1992.

[7] H.F.Engler, "Advanced technologies for ultra wideband system design," International Symposium on Electromagnetic Compatibility Symposium, vol.2, pp.250-253, Aug. 1993.

[8] M.Ressler, L.Happ, L.Nguyen, Ton Tuan, M.Bennett, "The Army Research Laboratory ultra-wide band test bed radars," IEEE International Radar Conference, pp.686$691,1995$.

[9] M. Z. Win, F. Ramirez-Mireles, R. A. Scholtz, M. A. Barnes, "Ultra-wide bandwidth (UWB) signal propagation for outdoor wireless communications," IEEE $47^{\text {th }}$ Vehicular Technology Conference, vol.1, pp.251 255, May 1997.
[10] M. Z. Win and Robert A. Scholtz, "On the robustness of ultra-wide bandwidth signals in dense multipath environments," IEEE Communications Letters, vol.2, no.2, pp.51- 53, Feb. 1998.

[11] AA. Saleh, RA.Valenzuela, "A statistical model for indoor multipath propagation," IEEE Journal on Selected Areas Communication, vol.5, no.2, pp.128-37, 1987.

[12] Li and Rusch, "Multiuser Detection In DS-CDMA UWB in the Home Environment" in IEEE Journal on selected area in Communications, vol. 20, No. 9, pp. 1701-1711, Dec 2002.

[13] J. Foerster, Q. Li, "Channel modeling subcommittee report final," IEEE P802.15 WG for WPANs Technical Report, 